\title{
Correction to: Is Ultimate Attribution Error Universal? Inter-group Attribution for Success and Failure among University Students in Delhi
}

\author{
Shreela Misra ${ }^{1}$ - Arvind Kumar Mishra ${ }^{1}$
}

Published online: 8 January 2020

(c) National Academy of Psychology (NAOP) India 2020

\section{Correction: Psychol Stud https://doi.org/10.1007/s12646-019-00503-4}

In the Abstract, the sixth line should read as "Results indicated that both General Caste category and Scheduled Caste category participants showed no inter-group attribution bias. These results are explained in terms of role of education in influencing the inter-group attribution process, suggesting that ultimate attribution error is not universal".

Under the Discussion section, in the first paragraph, the second line should read as "Similarly, the SC category students also did not demonstrate any inter-group attribution bias. These findings did not support the predictions based on the UAE hypothesis, proposed by Pettigrew (1979)".

Under the Discussion section, in the second paragraph, the sixth and seventh lines should read as "However, in the present study, both GC and SC category observers showed no inter-group attribution bias. These findings are contrary to predictions made and may be explained in terms of the role of education in influencing attribution".

In the Education and Attribution section, the first line should read as "The finding that there was no inter-group attribution bias in making attribution for success and failure is a departure from the existing literature".

The original article can be found online at https://doi.org/10.1007/s12646-019-00503-4.

Shreela Misra

shreela.preeti@gmail.com

Arvind Kumar Mishra

akmishra106@gmail.com

1 Jawaharlal Nehru University, New Delhi, India
In the Education and Attribution section, in the first paragraph, the 12th line should read as "These evidences suggest that higher education plays an important role in reducing prejudice and inter-group attribution bias in the minds of students belonging to both GC and SC categories, that is, dominant and subordinate castes alike".

In the Education and Attribution section, in the second paragraph, the third line should read as "At the same time, responses of students belonging to the discriminated and marginalized groups range from protests, 'dharnas,' and rallies to suicide in extreme cases".

In the Education and Attribution section, the third paragraph should read as follows:

Previous research in the field has mostly always demonstrated inter-group attribution bias and in certain cases also patterns of in-group derogation on the part of minority group members. For example, in the context of race, Hewstone, Wagner and Machleit (1989) found that Turkish students attributed in-group success more to good luck (external cause) than they did out-group success where the out-group was German students. Similarly, in-group derogating results have been reported in the context of gender as well (Deaux \& Emswiller 1974; Feather \& Simon 1975), suggesting that the minority or the subordinate groups tend to accept the dominant notion existing in the society. However, in the context of inter-group attribution for the positive and the negative events, out-group favoring attributions were reported only by Hewstone and Ward (1985). They found that, in Malaysia, the Chinese showed out-group favoritism. All these results do not support the hypothesis derived from social identity theory (SIT), suggesting a need to maintain positive distinctiveness of the self from the out-group. In the present study, students from the minority SC category did not demonstrate any bias be it in-group favoritism or out-group 
derogation. SIT argues that by virtue of belonging to different groups, people have a tendency to enhance in-group similarities and accentuate inter-group differences, thereby favoring their in-group relative to out-group. This is guided by the desire to maintain a positive self-esteem. By striving for a positive social identity, group members are motivated to think and act in ways that achieve or maintain a positive distinctiveness between the in-group and relevant out-groups (Hornsey, 2008). This was the process that was presumed to underpin real-world instances of inter-group differentiation and out-group derogation. To cite empirical research, it has been found that when group members demonstrate in-group favoritism, they are more likely to demonstrate higher social identity-based self-esteem rather than personal identitybased self-esteem (Hunter, Reid, Stokell \& Platow, 2000). Similarly, Hewstone (1990) pointed out that ethnocentric inter-group attributions establish positive social identity and that their function is to maintain positive social identity and collective self-esteem. Islam and Hewstone (1993) also demonstrated strong relationship between ethnocentric attributions and self-esteem. As can be seen, findings of the present study do not support this prediction of SIT. Moreover, the results also do not reflect the present occurring in higher education and educational institutions. The scenario of continuous agitations over the issue of reservations versus merit throughout the country is clearly not reflected by the findings of the study. Results demonstrate no differences in the attribution pattern for both groups of students. While social desirability effects may explain this pattern of attribution on the part of GC category students for the SC category students, revelation of their identities may explain the same. Acting as a part of a group allows individuals to stress upon social rather than personal identity, whereas the same is not possible in a one-to-one setup. However, the sensitivity of the groups under consideration as well as the procedure followed for data collection may have influenced the responses of students from SC category. Therefore, in order to explain the attribution made by the SC category students in the present study, it is imperative to probe deeper beyond these superficial results. The finding that both groups under study did not demonstrate any bias in making attributions is not only contrary to existing literature but also to the present scenario in the country. This raises questions regarding the role of ideology in the attribution process.

In the fourth paragraph, the fourth line should read as "This process of rationalization of the ideology of society has not been demonstrated by results of the present study".
The Contributions, Limitations and Directions for Future Research section should read as follows:

The study highlighted the distinction between intergroup attribution for positive and negative events and that for success and failure outcomes. In the context of intergroup attribution for success and failure outcomes, surprising results emerged which provide important insights into social cognition processes in Indian society. The dominant group of General Caste (GC) category students as well as the subordinate group of SC category students did not demonstrate any inter-group attribution bias. It may be that the GC and SC category students are aware of existing stereotypes and ideologies but are able to curb their prejudice due to social desirability effects. Therefore, it is necessary to further investigate these findings. Social desirability effects should be probed for students. Similarly, attempts should be made to understand their responses in more detail. For this purpose, qualitative study can be used. With a limited sample, it is not possible to generalize the results; therefore, research should be conducted with larger samples to make further generalizations. Another limitation is that students from other backward class (OBC) were not included in the study, which would have provided deeper insights into the complicated caste system. It is not a simple General versus SC category demarcation in India, and research should attempt to capture these dynamics as well. A methodological shortcoming of the study is that in the hypothetical situations used to measure attributions, participants responded to a list of causes presented by the researchers. These were then rated as internal/external, stable/unstable and controllable/uncontrollable by the researchers. Instead, Russell's Causal Dimension Scale (1982) could have been used to understand how participants attribute the causes of success and failure along these dimensions. The scale is widely used in interpersonal attribution research, and its incorporation in inter-group context will also provide beneficial uses. Finally, in addition to conducting quantitative research, it is necessary to validate it with qualitative tools like observation of student interactions and experiences in university campus. Survey and in-depth interviews can also be conducted to understand how students understand and attribute success and failure.

Publisher's Note Springer Nature remains neutral with regard to jurisdictional claims in published maps and institutional affiliations. 